\title{
Bidimensional Fuzzy Initial Value Problem of Autocorrelated Fuzzy Processes via Cross Product: The Prey-predator Model
}

\author{
${ }^{*}$ Beatriz Laiate $^{a}$ and Laécio C. Barros ${ }^{b}$ and Francielle Santo Pedro ${ }^{c}$ and Estevão Esmi ${ }^{d}$ \\ ${ }^{a}$ Department of Applied Mathematics, University of Campinas, Brazil, beatrizlaiate@gmail.com \\ ${ }^{b}$ Department of Applied Mathematics, University of Campinas, Brazil, laeciocb@ime.unicamp.br \\ ${ }^{c}$ Multidisciplinary Department, Federal University of São Paulo, Osasco, Brazil, fsimoes@unifesp.br \\ ${ }^{d}$ Department of Applied Mathematics, University of Campinas, Brazil, eelaureano@ime.unicamp.br
}

\begin{abstract}
This paper introduces the notion of a bidimensional fuzzy initial value problem for a special class of fuzzy functions. These functions, also called $A$-linearly correlated fuzzy processes, are a particular case of the socalled S-linearly correlated fuzzy processes, whose range is embedded in Banach spaces of fuzzy numbers. To this end, it recalls the notion of cross product and proves that this operation is the Zadeh's extension of the linearization of the real-valued function given by the product of two real numbers. The equivalence between the bidimensional FIVP under the Fréchet derivative and a nonlinear classical initial value problem is provided. Lastly, an application on the preypredator is presented.
\end{abstract}

Keywords: Fuzzy numbers, Strongly linearly independence, Autocorrelated fuzzy processes, Fréchet derivative, Cross product, Lotka-Volterra model.

\section{Introduction}

The prey-predator model and its generalizations have already been studied under the approach of fuzzy sets theory in various papers [7, 22, 23, 24, ?]. Population dynamics involving two or more species are classified according to the type of interaction between the species. The prey-predator fits in the specific case where the increasing of one of the species occurs with the decreasing of the another [21]. Lotka [20] and Volterra [27] introduced the simplest prey-predator system with interactions represented as molecular interactions, ruled by the mass-action principle.

Population dynamics as the malthusian growth, for instance, were studied in previous studies when consid- ered as autocorrelated processes [10]. Later, these processes were also called $A$-linearly correlated processes [19]. A theory of calculus based on the Fréchet derivative and Riemann integral for these fuzzy functions is already well established in the literature $[15,26]$. It is noteworthy the fact that an $A$-linearly correlated fuzzy process is also an autocorrelated fuzzy process and vice versa [17], for which a memory coefficient $\mathscr{F}_{t, h}$, represented by a linear function, characterizes the function as an interactive fuzzy process [25].

The usual arithmetic operations in the class of fuzzy numbers are given in terms of the Zadeh's extension principle $[4,8]$. Alternatively, the arithmetic operations between two fuzzy numbers can be defined from the joint possibility distribution betweeen them [11]. In particular, the usual arithmetic operations correspond to the case when the joint possibility distribution involved is given by the minimum. With the purpose of avoiding deal with joint possibility distributions, the concept of linearly correlation was introduced [10]. This concept, in turn, has raised from the notion of completely correlated fuzzy numbers, for which a detailed arithmetic is already well-established $[6,10]$.

The arithmetic operations studied in this paper are not based on the concept of joint possibility distribution. Instead, the sum and the difference between the fuzzy numbers involved are given as induced operations in Banach spaces of fuzzy numbers isomorphic to $\mathbb{R}^{2}$ [15]. The operation of product between the fuzzy numbers, called $\psi$-cross product, is a generalization of the concept of cross-product $[1,2,3]$, was introduced in [19] and extended in [18].

This paper is organized as follows: Section 2 presents concepts on fuzzy sets theory and, more specifically, the theory concerning the $A$-linearly correlated fuzzy processes. Section 3 establishes the cross-product as the Zadeh's extension as the linearization of a realvalued function and recalls the definition of $\psi$-cross product. Section 4 presents the Lotka-Volterra model 
considering the prey and the predator as $A$-linearly correlated fuzzy processes and provides some results considering two different scenarios. Lastly, Section 5 encloses the manuscript with some final remarks and perspectives for further studies.

\section{Mathematical Background}

A fuzzy number $A$ is a fuzzy subset of $\mathbb{R}$ with a normal, fuzzy convex and upper-semicontinuous membership function $A: \mathbb{R} \rightarrow[0,1]$, and additionally, with a compact support [10]. The class of fuzzy numbers is denoted by $\mathbb{R}_{\mathscr{F}}$.

For each $A \in \mathbb{R}_{\mathscr{F}}$, the $\alpha$-levels of $A$ are crisp subsets defined by

$$
[A]_{\alpha}=\left\{\begin{array}{l}
\{x \in \mathbb{R}: A(x) \geq \alpha\}, \alpha \in(0,1] \\
\overline{\{x \in \mathbb{R}: A(x)>0\}}, \alpha=0,
\end{array}\right.
$$

where $\bar{X}$ denotes the closure of $X \subset \mathbb{R}$.

If $A \in \mathbb{R}_{\mathscr{F}}$, then its $\alpha$-levels are compact intervals, that is, we can write $[A]_{\alpha}=\left[a_{\alpha}^{-}, a_{\alpha}^{+}\right]$, where $a_{\alpha}^{-}$and $a_{\alpha}^{+}$ denote the lower and upper endpoints of $[A]_{\alpha}$, respectively, for all $\alpha \in[0,1]$. For each $\alpha \in[0,1]$, the lenght of $[A]_{\alpha}$ is given by len $\left([A]_{\alpha}\right)=a_{\alpha}^{+}-a_{\alpha}^{-}$. The diameter of a fuzzy number $A$ is defined as the lenght of the 0 -level of $A$, that is, $\operatorname{diam}(A)=a_{0}^{+}-a_{0}^{-}$. The diameter of a fuzzy number can by associated as a measure of the uncertainty modeled by this number .

If $X$ and $Y$ are crisp sets, the Zadeh's extension principle of a function $f: X \rightarrow Y$ indicates the image of $f$ when applied to a fuzzy subset $A$ of $X$ [8]. Next, we enunciate the Zadeh's extension principle for functions defined specifically in $\mathbb{R}^{n}$.

Definition 1. [9, 28] Let $f: \mathbb{R}^{n} \rightarrow \mathbb{R}^{n}$ and $A \in \mathscr{F}\left(\mathbb{R}^{n}\right)$, where $\mathscr{F}\left(\mathbb{R}^{n}\right)$ denotes the class of all fuzzy subsets of $\mathbb{R}^{n}$. The Zadeh's extension of $f$ is the function $\hat{f}: \mathscr{F}\left(\mathbb{R}^{n}\right) \rightarrow \mathscr{F}\left(\mathbb{R}^{n}\right)$ whose membership function is given by

$$
\hat{f}(A)(x)=\left\{\begin{aligned}
\sup _{y \in f^{-1}(x)} A(y), & \text { if } f^{-1}(x) \neq \emptyset \\
0, & \text { if } f^{-1}(x)=\emptyset,
\end{aligned}\right.
$$

where $f^{-1}(x)=\left\{y \in \mathbb{R}^{n}: f(y)=x\right\}$.

The next theorem is a well-known result concerning the image of $\hat{f}$ whenever $f$ is continuous.

Theorem 1. [9] If $f: \mathbb{R}^{n} \rightarrow \mathbb{R}^{n}$ is continuous, then the Zadeh's extension of $f$ is well defined and

$$
[\hat{f}(A)]_{\alpha}=f\left([A]_{\alpha}\right)
$$

holds for all $\alpha \in[0,1]$.
The usual arithmetic operations in the class of fuzzy numbers are defined in terms of the Zadeh's extension principle. Specifically, if $B, C \in \mathbb{R}_{\mathscr{F}}$ are given levelwise by $[B]_{\alpha}=\left[b_{\alpha}^{-}, b_{\alpha}^{+}\right]$and $[C]_{\alpha}=\left[c_{\alpha}^{-}, c_{\alpha}^{+}\right]$, then

$$
\begin{aligned}
& {[B+C]_{\alpha}=\left[b_{\alpha}^{-}+c_{\alpha}^{-}, b_{\alpha}^{+}+c_{\alpha}^{+}\right]} \\
& {[B-C]_{\alpha}=\left[b_{\alpha}^{-}-c_{\alpha}^{+}, b_{\alpha}^{+}-c_{\alpha}^{-}\right]} \\
& {[\lambda B]_{\alpha}=\left\{\begin{array}{l}
{\left[\lambda b_{\alpha}^{-}, \lambda b_{\alpha}^{+}\right], \lambda \geq 0} \\
{\left[\lambda b_{\alpha}^{+}, \lambda b_{\alpha}^{-}\right], \lambda<0}
\end{array}\right.}
\end{aligned}
$$

for all $\alpha \in[0,1]$ and $\lambda \in \mathbb{R}[8]$.

Two fuzzy numbers $B$ and $C$ are said to by linearly correlated if there exist $q, r \in \mathbb{R}$ with $q \neq 0$ such that $B=q C+r$ or, equivalently, $[B]_{\alpha}=q[C]_{\alpha}+r$, for all $\alpha \in[0,1]$. For a given $A \in \mathbb{R}_{\mathscr{F}}$, the set of all fuzzy numbers linearly correlated to $A$ is given by

$\mathbb{R}_{\mathscr{F}(A)}=\left\{B \in \mathbb{R}_{\mathscr{F}}:[B]_{\alpha}=q[A]_{\alpha}+r, \forall \alpha \in[0,1]\right\}[15]$

Under some weak conditions, the space $\mathbb{R}_{\mathscr{F}(A)}$ is a Banach space of $\mathbb{R}_{\mathscr{F}}$ of dimension 2 [15]. These conditions are related to the symmetry of $A$, as we shall see next.

A fuzzy number $A$ is said to be symmetric with respect to $x \in \mathbb{R}$ (symmetric for short) if $A(x-y)=A(x+y)$ for all $y \in \mathbb{R}$. In this case, we denote $(A \mid x)$. We say that $A$ is non-symmetric if there is no $x$ such that $A$ is symmetric $[15,26]$. The next lemma characterizes a symmetric fuzzy number $A$ via its levels sets:

Lemma 1. [15, 26] A fuzzy number is symmetric with respect to $x \in \mathbb{R}$ if, and only if, $\delta_{A}(\alpha) \doteq a_{\alpha}^{-}+a_{\alpha}^{+}=2 x$, $\forall \alpha \in[0,1]$.

For a given subset $\left\{A_{1}, \ldots, A_{n}\right\}$ of $\mathbb{R}_{\mathscr{F}}$, the set $S\left(A_{1}, \ldots, A_{n}\right)$ denotes the set of all linear combinations of $A_{1}, \ldots, A_{n}$, that is,

$$
S\left(A_{1}, \ldots, A_{n}\right)=\left\{q_{1} A_{1}+\ldots+q_{n} A_{n} \mid q_{1}, \ldots, q_{n} \in \mathbb{R}\right\},
$$

where + and $q_{i} A_{i}$ stand for the usual sum and scalar multiplication in the class of fuzzy numbers [13].

The case concerning the symmetry with respect to 0 is of key importance, since it induces the concept of strongly linearly independence given by the next definition.

Definition 2. [13] A subset $\left\{A_{1}, \ldots, A_{n}\right\} \subset \mathbb{R}_{\mathscr{F}}$ is said to be strongly linearly independent (SLIfor short) iffor all $A \in S\left(A_{1}, \ldots, A_{n}\right)$ such that $A=q_{1} A_{1}+\ldots+q_{n} A_{n}$, the following implication holds:

$$
(A \mid 0) \Rightarrow q_{1}=\ldots=q_{n}=0 .
$$

The next theorem characterizes the SLI sets of fuzzy numbers via an isomorphism between $\mathbb{R}^{n}$ and the set $S\left(A_{1}, \ldots, A_{n}\right)$. 
Theorem 2. [13] The set $\left\{A_{1}, \ldots, A_{n}\right\} \subset \mathbb{R}_{\mathscr{F}}$ is SLI if, and only if, the function $\psi: \mathbb{R}^{n} \rightarrow S\left(A_{1}, \ldots, A_{n}\right)$ given by

$$
\psi\left(x_{1}, \ldots, x_{n}\right)=x_{1} A_{1}+\ldots+x_{n} A_{n}
$$

is a bijection.

The concept of strongly linearly independence of a set of fuzzy numbers resembles the concept of linear independence of a set in a vector space. In fact, a set of fuzzy numbers $\left\{A_{1}, \ldots, A_{n}\right\}$ is SLI if, and only if, the set of functions $\left\{\delta_{A_{1}}(\alpha), \ldots, \delta_{A_{n}}(\alpha)\right\}$ is linearly independent in $\mathscr{B}([0,1], \mathbb{R})$, the set of all bounded realvalued functions defined in $[0,1][13]$.

From the bijectiveness of $\psi: \mathbb{R}^{n} \rightarrow S\left(A_{1}, \ldots, A_{n}\right)$ we can define induced operations of sum and scalar multiplication in $S\left(A_{1}, \ldots, A_{n}\right)$ whenever $\left\{A_{1}, \ldots, A_{n}\right\}$ is an SLI set, namely

$$
B+{ }_{\psi} C \doteq \psi\left(\psi^{-1}(B)+\psi^{-1}(C)\right)
$$

and

$$
\lambda \cdot{ }_{\psi} B \doteq \psi\left(\lambda \psi^{-1}(B)\right)
$$

for all $B, C \in S\left(A_{1}, \ldots, A_{n}\right)$ and $\lambda \in \mathbb{R}$. That means that if $B=p_{1} A_{1}+\ldots+p_{n} A_{n}$ and $C=q_{1} A_{1}+\ldots+q_{n} A_{n}$, then $B+{ }_{\psi} C=\left(p_{1}+q_{1}\right) A_{1}+\ldots+\left(p_{n}+q_{n}\right) A_{n}$. Similarly, the induced difference between $B$ and $C$ is given by $B-{ }_{\psi} C \doteq B+(-1) \cdot{ }_{\psi} C$. Also, we may define an induced norm $\|\cdot\|_{\psi}: S\left(A_{1}, \ldots, A_{n}\right) \rightarrow[0, \infty)$ given by $\|B\|_{\psi} \doteq\left\|\psi^{-1}(B)\right\|_{\infty}, \forall B \in S\left(A_{1}, \ldots, A_{n}\right)$, where $\left\|\left(p_{1}, \ldots, p_{n}\right)\right\|_{\infty}=\max _{i=1, \ldots, n}\left|p_{i}\right|$, for all $\left(p_{1}, \ldots, p_{n}\right) \in \mathbb{R}^{n}$ $[13,14]$.

The next corollary is an immediate consequence of Theorem 2 and from the fact that $\left(\mathbb{R}^{n},\|\cdot\|_{\infty}\right)$ is a Banach space [16]:

Corollary 1. [13] Let $\left\{A_{1}, \ldots, A_{n}\right\}$ be SLI. Then $\left(S\left(A_{1}, \ldots, A_{n}\right),{ }_{\psi}, \cdot{ },\|\cdot\|_{\psi}\right)$ is a Banach space.

In this manuscript we deal with the case where the SLI set involved is given by $\left\{A_{1}, A_{2}\right\}=\{A, 1\}$. Note that by Theorem 2, $\{A, 1\}$ is SLI if, and only if, $A \in \mathbb{R}_{\mathscr{F}}$ is nonsymmetric. In fact, the set $\left\{\delta_{A}, \delta_{\chi_{\{1\}}}\right\}=\left\{a_{\alpha}^{-}+a_{\alpha}^{+}, 2\right\}$ is linearly independent if, and only if, $a_{\alpha}^{-}+a_{\alpha}^{+}$is not constant for all $\alpha \in[0,1]$. By Lemma 1 , this condition holds if, and only if, $A \in \mathbb{R}_{\mathscr{F}}$ is non-symmetric. In this case, $S(A, 1)=\mathbb{R}_{\mathscr{F}(A)}$, the class of all $A$-linearly correlated fuzzy numbers, is isomorphic to $\mathbb{R}^{2}$. According to Corollary $1,\left(S(A, 1),+_{\psi},{ }^{\cdot} \psi,\|\cdot\|_{\psi}\right)$ is a Banach space [15].

The induced sum between two A-linearly correlated fuzzy numbers always produce a fuzzy number whose diameter do not exceedes the diameter of the usual sum in $\mathbb{R}_{\mathscr{F}}$, that is, $B+{ }_{\psi} C \subseteq B+C, \forall B, C \in \mathbb{R}_{\mathscr{F}(A)}$. In particular, $\lambda \cdot{ }_{\psi} B=\lambda B, \forall \lambda \in \mathbb{R}$ holds [14, 15]. For this reason, we henceforth omit the notation $\cdot \psi$.

In order to study fuzzy differential equations for functions defined in Banach spaces, let us consider $A \in \mathbb{R}_{\mathscr{F}}$ non-symmetric. An A-linearly correlated fuzzy process is a fuzzy number-valued function of the type $f:[a, b] \rightarrow S(A, 1)$, that is,

$$
f(t)=q(t) A+r(t),
$$

where $q, r:[a, b] \rightarrow \mathbb{R}$. Moreover, if $f$ is an $A$-linearly correlated fuzzy process, then we can write $f(t)=$ $\psi(q(t), r(t))$ for all $t \in[a, b]$. Moreover, if $f$ is an A-linearly correlated fuzzy process, then it is also an autocorrelated process, that is, for $0<|h|$ sufficiently small, there are $q(h), r(h) \in \mathbb{R}$ such that

$$
f(t+h)=q(h) f(t)+r(h)
$$

for all $t \in[a, b][10,17]$. The pair $(q(h), r(h))$ can be seen as a memory coefficient, that is, $f(t+h)=$ $\mathscr{F}_{t, h}(f(t))$, which can be used to modelling some kinds of population dynamics [25].

The Fréchet derivative of $A$-linearly correlated fuzzy processes is well defined as established in $[15,26]$. Analogously to the classical theory, we say that $f$ : $[a, b] \rightarrow S(A, 1)$ is Fréchet differentiable at $t \in[a, b](\mathrm{F}-$ differentiable for short) if there exists a continuous linear operator $f^{\prime}[t]: \mathbb{R} \rightarrow S(A, 1)$ such that

$$
f(t+h)=f(t)+{ }_{\psi} f^{\prime}[t](h)+{ }_{\psi} \omega(h),
$$

with $\lim _{h \rightarrow 0} \frac{\|\omega(h)\|_{\psi}}{|h|}=0$ [14]. As well as in the classical case, the expression given by Equation (3) is called the Fréchet expansion of $f$ at $t$ [5].

Theorem 3. (Adapted from $[14,15])$ Let $A \in \mathbb{R}_{\mathscr{F}}$ be non-symmetric and $f:[a, b] \longrightarrow S(A, 1)$ be such that $f(t)=q(t) A_{1}+r$ for all $t \in[a, b]$. The function $f$ is Fréchet differentiable at $t \in[a, b]$ if, and only if, $q, r$ : $[a, b] \longrightarrow \mathbb{R}$ are differentiable at $t$. Additionally,

$$
f^{\prime}[t](h)=q^{\prime}(t) A h+r^{\prime}(t) h
$$

for all $h \in \mathbb{R}$.

Definition 3. (Adapted from $[14,15]$ ) Let $A \in \mathbb{R}_{\mathscr{F}}$ be non-symmetric. The A-linearly correlated fuzzy process $f:[a, b] \longrightarrow S(A, 1)$ is said to be $\psi$-differentiable at $t \in[a, b]$ if there exists a fuzzy number $f^{\prime}(t) \in S(A, 1)$ such that

$$
\lim _{h \rightarrow 0} \frac{1}{h} \cdot \psi\left(f(t+h)-{ }_{\psi} f(t)\right)=f^{\prime}(t) .
$$

In this case, we say that $f^{\prime}(t)$ is the $\psi$-derivative of $f$ at $t$. Moreover, $f$ is said to be $\psi$-differentiable in $[a, b]$ if $f^{\prime}(t)$ exists for all $t \in[a, b]$. 
Theorem 4. (Adapted from [14]) Let $A \in \mathbb{R}_{\mathscr{F}}$ be nonsymmetric. The A-linearly correlated fuzzy process $f$ : $[a, b] \longrightarrow S(A, 1)$ is $\psi$-differentiable at $t \in[a, b]$ if, and only if, $f$ is F-differentiable at $t$. Moreover, we have that

$$
f^{\prime}[t](1)=f^{\prime}(t) .
$$

Corollary 2. (Adapted from $[14,15])$ Let $A \in \mathbb{R}_{\mathscr{F}}$ be non-symmetric and $f:[a, b] \longrightarrow S(A, 1)$ such that $f(t)=q(t) A+r(t)$ for all $t \in[a, b]$. The A-linearly correlated fuzzy process $f$ is $\psi$-differentiable at $t \in[a, b]$ if, and only if, $q, r:[a, b] \longrightarrow \mathbb{R}^{n}$ are differentiable at $t$. Additionally,

$$
f^{\prime}(t)=q^{\prime}(t) A+r^{\prime}(t)
$$

Provided that the induced operations of addition and subtraction are well defined in $S(A, 1)$, the operation of product of $A$-linearly correlated fuzzy numbers is presented in the next section. This operation was introduced in [19] and extended in [18] and it is based on the concept of cross product, set for fuzzy numbers in general.

\section{Cross product of fuzzy numbers and its generalizations}

A fuzzy number $W$ is said to be positive (strictly positive, resp.) if $w_{1}^{-} \geq 0\left(w_{1}^{-}>0\right.$, resp. $)$ and negative (strictly negative, resp.) if $w_{1}^{+} \leq 0\left(w_{1}^{+}<0\right.$, resp.). The set of all positive or negative fuzzy numbers is denoted by

$$
\mathbb{R}_{\mathscr{F}}^{*}=\left\{W \in \mathbb{R}_{\mathscr{F}}: 0 \notin \operatorname{int}\left(w_{1}\right)\right\},
$$

where int $\left(w_{1}\right)$ denotes the interior of the core of $W$ [4].

Proposition 1. [1, 2, 3] If $B$ and $C$ are positive fuzzy numbers, then $W=B \odot C$ defined by $[W]_{\alpha}=\left[w_{\alpha}^{-}, w_{\alpha}^{+}\right]$, where

$$
w_{\alpha}^{-}=b_{\alpha}^{-} c_{1}^{-}+b_{1}^{-} c_{\alpha}^{-}-b_{1}^{-} c_{1}^{-}
$$

and

$$
w_{\alpha}^{+}=b_{\alpha}^{+} c_{1}^{+}+b_{1}^{+} c_{\alpha}^{+}-b_{1}^{+} c_{1}^{+}
$$

for every $\alpha \in[0,1]$, is a positive fuzzy number.

Proposition 2. [1, 4] Let $u$ and $v$ be two fuzzy numbers.

i) If $B$ is positive and $C$ is negative, then

$$
B \odot C=-(B \odot(-C))
$$

is a negative fuzzy number;

ii) If $B$ is negative and $C$ is positive, then

$$
B \odot C=-((-B) \odot C)
$$

is negative fuzzy number; iii) If $B$ and $C$ are negative, then

$$
B \odot C=(-B) \odot(-C)
$$

is a positive fuzzy number.

Definition 4. [3] The binary operation on $\mathbb{R}_{\mathscr{F}}^{*}$ given by Proposition 1 is called the cross product of fuzzy numbers.

The cross product is defined for any fuzzy numbers whose core contains only one element, that is, for any fuzzy numbers in

$\mathbb{R}_{\mathscr{F}}^{\wedge}=\left\{B \in \mathbb{R}_{\mathscr{F}}^{*}:[B]_{1}\right.$ contains only one element $\}[4]$.

Proposition 2 ensures that if $B, C \in \mathbb{R}_{\mathscr{F}}$ and $W=$ $B \odot C$, then we can write, levelwise, $[W]_{\alpha}=c_{1}[B]_{\alpha}+$ $b_{1}[C]_{\alpha}-b_{1} c_{1}$ for all $\alpha \in[0,1]$, that is,

$$
W=c_{1} B+b_{1} C-b_{1} c_{1} .
$$

holds.

It is well known that the usual multiplication of two fuzzy numbers is given in terms of Zadeh's extension principle. This means that for all $B, C \in \mathbb{R}_{\mathscr{F}}$, we have that $(B . C)(z)=\hat{f}(A, B)(z), \forall z \in \mathbb{R}$, where $f(x, y)=x y$ for all $(x, y) \in \mathbb{R}^{2}$. Let $P: \mathbb{R}^{2} \rightarrow \mathbb{R}$ be the linearization of $f$ around $\left(x_{0}, y_{0}\right)$, that is,

$$
\begin{aligned}
P(x, y) & =f\left(x_{0}, y_{0}\right)+\frac{\partial f}{\partial x}\left(x_{0}, y_{0}\right)\left(x-x_{0}\right)+\frac{\partial f}{\partial y}\left(x_{0}, y_{0}\right)\left(y-y_{0}\right) \\
& =x_{0} y_{0}+y_{0}\left(x-x_{0}\right)+x_{0}\left(y-y_{0}\right) \\
& =x_{0} y+y_{0} x-x_{0} y_{0} .
\end{aligned}
$$

The next proposition establishes a relation between Definition 4 and Equation (7).

Proposition 3. Let $B, C \in \mathbb{R}_{\mathscr{F}}^{\wedge}$ with $[B]_{1}=\left\{b_{1}\right\}$ and $[C]_{1}=\left\{c_{1}\right\}$. The cross product between $B$ and $C$ is the Zadeh's extension of the linearization of $f(x, y)=x y$ around $\left(b_{1}, c_{1}\right)$, that is,

$$
B \odot C=\hat{P}(B, C),
$$

where $P: \mathbb{R}^{2} \rightarrow \mathbb{R}$ is given as Equation (7) for $\left(x_{0}, y_{0}\right)=\left(b_{1}, c_{1}\right)$.

Proof. The function $P(x, y)=b_{1} y+c_{1} x-b_{1} c_{1}$ is continuous in $\mathbb{R}^{2}$. By Theorem 1 , if $\hat{P}: \mathbb{R}_{\mathscr{F}} \times \mathbb{R}_{\mathscr{F}} \rightarrow \mathbb{R}_{\mathscr{F}}$ is the Zadeh's extension of $P: \mathbb{R}^{2} \rightarrow \mathbb{R}$, then

$$
\hat{P}\left([B]_{\alpha},[C]_{\alpha}\right)=b_{1}[C]_{\alpha}+c_{1}[B]_{\alpha}-b_{1} c_{1}
$$

holds for all $\alpha \in[0,1]$, that is, $\hat{P}(B, C)=B \odot C$, for all $B, C \in \mathbb{R}_{\mathscr{F}}$. 
If $B$ and $C$ are $A$-linearly correlated fuzzy numbers for some $A \in \mathbb{R}_{\mathscr{F}}$ non-symmetric, then $B \odot C$ is not an $A$ linearly correlated fuzzy number in general [19]. This shortcoming can be overcome by the following definition:

Definition 5. (Adapted from $[18,19])$ Let $A \in \mathbb{R}_{\mathscr{F}}^{\wedge}$ be non-symmetric and $B, C \in S(A, 1)$. The $\psi$-cross product between $B$ and $C$ is defined as the fuzzy number $W$ given by

$$
W=B \odot_{\psi} C \doteq c_{1} B+{ }_{\psi} b_{1} C-{ }_{\psi} b_{1} c_{1},
$$

where $+_{\psi}(-\psi)$ stands for the induced sum (subtraction) in $S(A, 1)$.

Definition 5 establishes an operation of product between linearly correlated fuzzy numbers that resembles the concept of cross product. They differ only by the induced operations of sum and subtraction, from which we conclude that the space $S(A, 1)$ is closed under the $\psi$-cross product whenever $A \in \mathbb{R}_{\mathscr{F}}$ is non-symmetric [19]. Theorem 5 establishes some algebraic properties of the operation $\odot \psi$.

Theorem 5. (Adapted from $[18,19])$ Let $A \in \mathbb{R}_{\mathscr{F}}^{\wedge}$ be non-symmetric, $B, C, D \in S(A, 1)$ and $\lambda \in \mathbb{R}$. The following properties hold true:

i) $B \odot{ }_{\psi} C=C \odot_{\psi} B$;

ii) $\left(B+{ }_{\psi} C\right) \odot{ }_{\psi} D=\left(B \odot{ }_{\psi} D\right)+{ }_{\psi}\left(C \odot{ }_{\psi} D\right)$;

iii) $(\lambda B) \odot \psi{ }_{\psi} C=\lambda\left(B \odot{ }_{\psi} C\right)$;

iv) $\left(B \odot_{\psi} C\right) \odot_{\psi} D=B \odot_{\psi}\left(C \odot_{\psi} D\right)$;

v) $B \odot_{\psi} 1=1 \odot_{\psi} B=B$, where $1 \in \mathbb{R}_{\mathscr{F}}$ is regarded as a singleton;

vi) $B \odot{ }_{\psi} C \subseteq B \odot C$;

It is worth mentioning that the diameter of $B \odot{ }_{\psi} C$ can be writen explicitly in terms of the diameter of $A \in \mathbb{R}_{\mathscr{F}}$ whenever $B, C \in S(A, 1)$, namely:

$$
\begin{aligned}
\operatorname{diam}\left(B \odot{ }_{\psi} C\right) & =\left|c_{1} q_{1}+b_{1} q_{2}\right| \operatorname{diam}(A) \\
& \leq\left|c_{1}\right| \operatorname{diam}(B)+\left|b_{1}\right| \operatorname{diam}(C) \\
& =\operatorname{diam}(B \odot C),
\end{aligned}
$$

where $B=q_{1} A+r_{1}, \quad C=q_{2} A+r_{2}$ for some $\left(q_{1}, r_{1}\right),\left(q_{2}, r_{2}\right) \in \mathbb{R}^{2}$ and $[B]_{1}=\left\{b_{1}\right\},[C]_{1}=\left\{c_{1}\right\}$. Equation (9) provides an alternative proof of item $v i$ ) of Theorem 5 in relation to that presented in $[18,19]$.

\section{The Lotka-Volterra model}

\subsection{Prey-predator system}

In this section we focus on the autonomous bidimensional FIVP for an $A$-linearly correlated fuzzy process, which is given by

$$
\left\{\begin{array}{l}
x^{\prime}(t)=f(x(t), y(t)) \\
y^{\prime}(t)=g(x(t), y(t))
\end{array}\right.
$$

where the initial conditions $x(0), y(0) \in S(A, 1)$ for some $A \in \mathbb{R}_{\mathscr{F}}^{\wedge}$ non-symmetric. We are assuming that $f, g: S(a, 1) \times S(A, 1) \rightarrow S(A, 1)$ are continuous w.r.t. the induced norm $\|\cdot\|_{\psi}$. Moreover, the solutions of the FIVP (10) are $A$-linearly correlated fuzzy processes $x, y:[0, T] \rightarrow S(A, 1)$ that satisfies (10) and the initial conditions given by $x(0)=q_{1}(0) A+r_{1}(0)=q_{1,0} A+$ $r_{1,0}$ and $y(0)=q_{2}(0) A+r_{2}(0)=q_{2,0} A+r_{2,0}$.

Let us assume that $f(x(t), y(t))=q_{f}(t) A+r_{f}(t)$ and $g(x(t), y(t))=q_{g}(t) A+r_{g}(t)$ for some $q_{f}, r_{f}, q_{g}, r_{g}$ : $[0, T] \rightarrow \mathbb{R}$. Since $x(t)=q_{1}(t) A+r_{1}(t)$ and $y(t)=$ $q_{2}(t) A+r_{2}(t)$ for some $q_{i}, r_{i}:[0, T] \rightarrow \mathbb{R}, i=1,2$, Equation (10) can be rewritten as

$$
\left\{\begin{array}{l}
q_{1}^{\prime}(t) A+r_{1}^{\prime}(t)=q_{f}(t) A+r_{f}(t) \\
q_{2}^{\prime}(t) A+r_{2}^{\prime}(t)=q_{g}(t) A+r_{g}(t)
\end{array}\right.
$$

or, equivalently,

$$
\left\{\begin{array}{l}
\psi\left(q_{1}^{\prime}(t), r_{1}^{\prime}(t)\right)=\psi\left(q_{f}(t), r_{f}(t)\right) \\
\psi\left(q_{2}^{\prime}(t), r_{2}^{\prime}(t)\right)=\psi\left(q_{g}(t), r_{g}(t)\right)
\end{array}\right.
$$

for all $t \in[0, T]$. From the bijectiveness of $\psi$, we have that Equation (12) is equivalent to the first-order classical system

$$
\left\{\begin{array}{l}
q_{1}^{\prime}(t)=q_{f}(t) \\
r_{1}^{\prime}(t)=r_{f}(t) \\
q_{2}^{\prime}(t)=q_{g}(t) \\
r_{2}^{\prime}(t)=r_{g}(t) .
\end{array}\right.
$$

with initial conditions given by $q_{i}(0)=q_{i, 0}$ and $r_{i}(0)=$ $r_{i, 0}, i=1,2$.

When in a two-especies system the growth rate of one population is decreased and the other increased, the populations are in a predator-prey system [21]. Originally, the prey-predator models, also called LotkaVolterra systems, were proposed by Volterra (1926) [27] to explain the oscilatory levels of certains fishes in the Adriatic sea, and Lotka [20], which proposed an study concerning chemical reactions with oscilatory behaviour. The simplest model is based on four simplyfing assumptions concerning the population dynamics [12]:

1. Prew grow in an unlimited way when predators do not keep then under control;

2. Predators depend on the presence of their prey to survive; 
3. The rate of predation depends on the likelihood that a prey is encountered by a predator;

4. The growth rate of the predator population is proportional to the rate of predation.

If $x=x(t)$ and $y=y(t)$ represent the population of preys and predators at the instant $t$, respectively, then the assumptions above give rise to the following system:

$$
\left\{\begin{array}{l}
x^{\prime}(t)=\delta x(t)-\alpha x(t) y(t) \\
y^{\prime}(t)=-\gamma y(t)+\beta x(t) y(t),
\end{array}\right.
$$

where $\delta$ is the net growth rate of the prey population without predation, $\gamma$ is the net death rate of the predators in the absence of prey and $\frac{\alpha}{\beta}$ represent the efficiency of the predation. The nonlinear term represents the meeting of the two species and it is based on the mass action law established for molecular collisions.

The prey-predator model for $A$-linearly correlated fuzzy processes under the $\psi$-derivative and the $\psi$-cross product is given by

$$
\left\{\begin{array}{l}
x^{\prime}(t)=\delta x(t)-{ }_{\psi} \alpha(x(t) \odot \psi y(t)) \\
y^{\prime}(t)=-\lambda y(t)+{ }_{\psi} \beta(x(t) \odot \psi y(t))
\end{array}\right.
$$

where the initial conditions are given by $A$-linearly correlated fuzzy numbers $x(0)=q_{1,0} A+r_{1,0}$ and $y(0)=$ $q_{2,0} A+r_{2,0}$. Let us denote $[x(t)]_{1}=\left\{x_{1}(t)\right\}$ and $[y(t)]_{1}=\left\{y_{1}(t)\right\}$ for all $t \geq 0$. By Definition 5, Equation (15) can be rewritten as

$\left\{\begin{array}{l}x^{\prime}(t)=\delta x(t)-{ }_{\psi} \alpha\left(y_{1}(t) x(t)+{ }_{\psi} x_{1}(t) y(t)-{ }_{\psi} x_{1}(t) y_{1}(t)\right) \\ y^{\prime}(t)=-\lambda y(t)+{ }_{\psi} \beta\left(y_{1}(t) x(t)+{ }_{\psi} x_{1}(t) y(t)-{ }_{\psi} x_{1}(t) y_{1}(t)\right)\end{array}\right.$

If we denote $[A]_{1}=\left\{a_{1}\right\}$, the FIVP represented by Equation (16) boils down to the nonlinear system of differential equations

$\left\{\begin{array}{l}q_{1}^{\prime}(t)=\delta q_{1}(t)-2 \alpha q_{1}(t) q_{2}(t) a_{1}-\alpha q_{1}(t) r_{2}(t)-\alpha r_{1}(t) q_{2}(t) \\ r_{1}^{\prime}(t)=\delta r_{1}(t)+\left(-\alpha a_{1}-a_{1}\right) r_{1}(t) q_{2}(t)+(-2 \alpha-1) r_{1}(t) r_{2}(t) \\ -\left(\alpha a_{1}+a_{1}\right) q_{1}(t) r_{2}(t)-a_{1}^{2} q_{1}(t) q_{2}(t) \\ q_{2}^{\prime}(t)=2 \beta q_{1}(t) q_{2}(t) a_{1}+\beta q_{1}(t) r_{2}(t)+\beta r_{1}(t) q_{2}(t)-\lambda q_{2}(t) \\ r_{2}^{\prime}(t)=2 \beta r_{1}(t) r_{2}(t)+\beta q_{1}(t) r_{2}(t) a_{1}+\beta r_{1}(t) q_{2}(t) a_{1}-\lambda r_{2}(t),\end{array}\right.$

whose initial conditions are given by $q_{i}(0)=q_{i, 0}$, $r_{i}(0)=r_{i, 0} \in \mathbb{R}$ for $i=1,2$.

\subsection{Results}

The oscilatory behaviour of the solutions of preypradator systems is well-known $[12,21]$. This is also observed when the populations are given by $A$-linearly correlated fuzzy processes. We considered two different scenarios: the first one is given by the case where the parameters of Equation (15) assume the values $\delta=0.15, \alpha=0.1, \gamma=0.05, \beta=0.08$. This case is depicted in Fig. 1. The second case is given by a high efficiency predation rate with a low net growth rate of the prey population, for which we assume $\delta=0.15$, $\alpha=0.1, \beta=0.08, \gamma=0.05$. This case is depicted in Fig. 2. Note that this scenario describes the extintion of the prey population, as well as the vanishing of the fuzziness of the solution.

In both cases we considered $A=(0 ; 1 ; 1.5)$ and initial conditions given by the $A$-linearly correlated fuzzy numbers $x(0)=1 A+0.5=(0.5 ; 1.5 ; 2)$ and $y(0)=$ $0.6 A+0.1=(0.1 ; 0.7 ; 1)$. The gray-scale represents the $\alpha$-levels of the solutions, where $\alpha$ varies from 0 to 1 . Moreover, the classical solutions coincide with the core of the fuzzy solutions whenever the initial conditions are given by $x_{0}=q_{1,0} a_{1}+r_{1}=1.5$ and $y_{0}=q_{2,0} a_{1}+r_{2}=0.7$. Thus, the fuzzy solutions of the FIVP (15) consist on an extension of the classical solutions of the IVP (14) whenever the classical initial conditions coincide with the core of the fuzzy initial conditions.
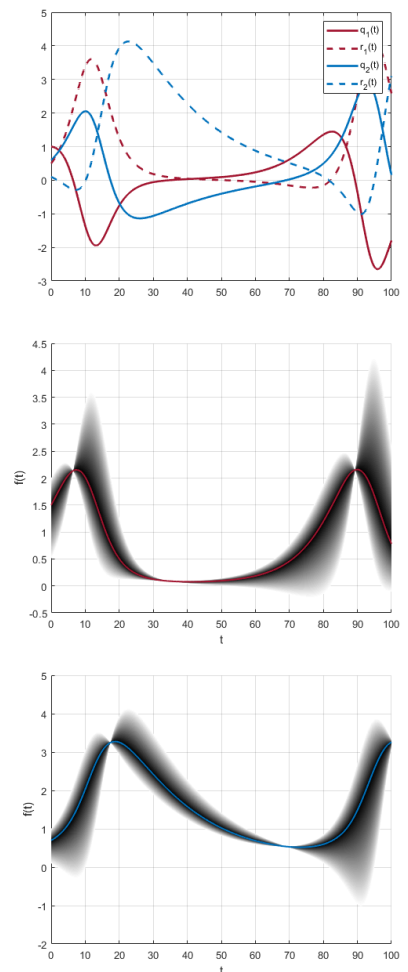

Figure 1: From top to bottom: depiction of the solutions of the IVP (17), where the filled (resp. dashed) red and blue lines represent $q_{1}(\cdot)$ and $q_{2}(\cdot)$ (resp. $r_{1}(\cdot)$ and $\left.r_{2}(\cdot)\right)$. In gray-scale, the numerical solutions of the FIVP (15) for $\delta=0.15, \alpha=0.1, \gamma=0.05, \beta=0.08$. The red and the blue line represent the classical solutions for the prey and predator populations, respectively. 

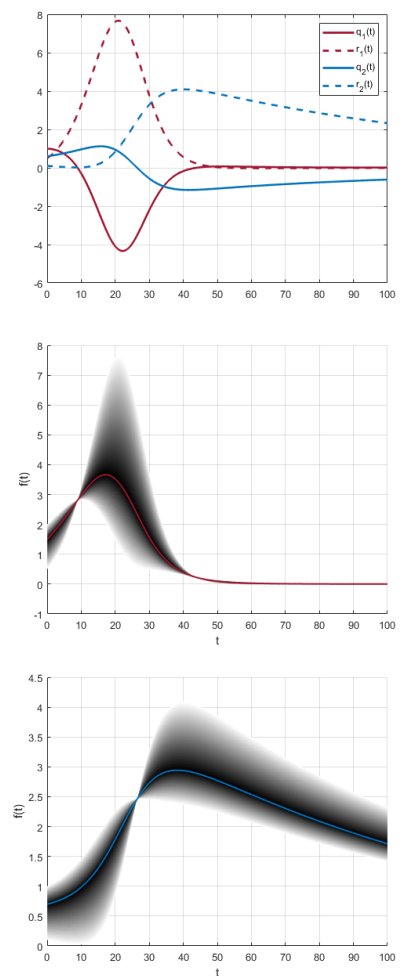

Figure 2: From top to bottom: depiction of the solutions of the IVP (17), where the filled (resp. dashed) red and blue lines represent $q_{1}(\cdot)$ and $q_{2}(\cdot)$ (resp. $r_{1}(\cdot)$ and $\left.r_{2}(\cdot)\right)$. In gray-scale, the numerical solutions of the FIVP (15) for $\delta=0.15, \alpha=0.1, \beta=0.02$ and $\gamma=0.01$. The red and the blue line represent the classical solutions for the prey and predator populations, respectively.

\section{Final comments}

In this paper, we recalled the concept of strongly linearly independent sets of fuzzy numbers and the Banach spaces generated by linear combinations of SLI fuzzy numbers. We considered, specifically, the set of all fuzzy numbers linearly correlated to a nonsymmetric fuzzy number $A[10,15]$. We also recalled the concept of $\psi$-cross product defined for $A$-linearly correlated fuzzy numbers introduced in $[18,19]$, which is based on the concept of cross product $[1,2,3]$, defined for fuzzy numbers in general.

In turn, we proved that the cross product between the fuzzy numbers $B$ and $C$ has proved to be the Zadeh's extension of the linearization of $f$ around $[B]_{1}$ and $[C]_{1}$, that is, $B \odot C=\hat{P}(B, C)$, where $\left.P(x, y)\right|_{\left(x_{0}, y_{0}\right)}=y_{0} x+$ $x_{0} y-x_{0} y_{0}$

The Lotka-Volterra model was studied when considering the prey and predator populations as $A$-linearly correlated fuzzy processes. To this end, the FIVP was given in terms of the $\psi$-derivative and the $\psi$-cross product. Two different scenarios were considered with the same initial conditions. In the first one, as in the classical case, an oscilatory behaviour of the solution was presented, while in the second one the prey population vanishes with time, as well as the fuzziness of the solution. In both cases the bigger the growth of the populations, the bigger the fuzziness of the solutions. In addition, the classical solutions coincide with the core of the fuzzy solutions whenever the initial conditions coincide with the core of the fuzzy initial conditions.

As the main contribution of the paper, we presented a mathematical tool to solve a bidimensional fuzzy initial value problem with classical calculus theory.

For further studies, we aim to establish the $\psi$-cross product as an interactive product between two fuzzy numbers, that is, as an arithmetic operation given by a joint possibility distribution previously defined. The stability analysis of the nonlinear classical systems corresponding to the bidimensional fuzzy systems under this product operation shall also be studied.

\section{Authors Contribution}

Beatriz Laiate conceived the presented idea, performed the computations, verified the analytical method, performed the analysis and wrote the manuscript. Beatriz Laiate, Laécio C. Barros, Francielle Santo Pedro and Estevão Esmi developed the theory and Laécio C. Barros, Francielle Santo Pedro and Estevão Esmi supervised the findings of the work. All authors discussed the results and contributed to the final manuscript.

\section{Acknowledgement}

This research was partially supported by thank $\mathrm{CNPq}$ grantee nos. 306546/2017-5 and 142309/2019-2, and Fapesp 2016/26040-7.

\section{References}

[1] A. Ban, B. Bede, Power series of fuzzy numbers with cross product and applications to fuzzy differential equations., Journal of Concrete \& Applicable Mathematics 4 (2).

[2] A. Ban, B. Bede, Properties of the cross product of fuzzy numbers, Journal of Fuzzy Mathematics 14 (3) (2006) 513.

[3] A. I. Ban, B. Bede, Cross product of 1-r fuzzy numbers and applications, Anal. Univ. Oradea, fasc. math 9 (2002) 95-108. 
[4] B. Bede, Mathematics of Fuzzy Sets and Fuzzy Logic, Vol. 295 of Studies in Fuzziness and Soft Computing, Springer, 2013.

[5] M. S. Berger, Nonlinearity and functional analysis: lectures on nonlinear problems in mathematical analysis, Academic press, 1977.

[6] C. Carlsson, R. Fullér, et al., Additions of completely correlated fuzzy numbers, in: IEEE International Conference on Fuzzy Systems, Vol. 1, IEEE, 2004, pp. 535-539.

[7] M. da Silva Peixoto, L. C. de Barros, R. C. Bassanezi, Predator-prey fuzzy model, Ecological Modelling 214 (1) (2008) 39-44.

[8] L. C. De Barros, R. Bassanezi, W. Lodwick, First Course in Fuzzy Logic, Fuzzy Dynamical Systems, and Biomathematics, Springer, 2016.

[9] L. C. de Barros, R. C. Bassanezi, P. A. Tonelli, On the continuity of the zadeh's extension, in: Proc. Seventh IFSA World Congress, Vol. 2, 1997, pp. 3-8.

[10] L. C. de Barros, F. S. Pedro, Fuzzy differential equations with interactive derivative, Fuzzy Sets and Systems 309 (2017) $64-80$.

[11] D. Dubois, H. Prade, Additions of interactive fuzzy numbers, IEEE Transactions on Automatic Control 26 (4) (1981) 926-936.

[12] L. Edelstein-Keshet, Mathematical models in biology, SIAM, 2005.

[13] E. Esmi, L. C. de Barros, F. Santo Pedro, B. Laiate, Banach spaces generated by strongly linearly independent fuzzy numbers, Fuzzy Sets and Systems.

[14] E. Esmi, B. Laiate, F. Santo Pedro, L. C. d. Barros, Calculus for fuzzy functions with fuzzy coefficients, Fuzzy Sets and Systems(submitted).

[15] E. Esmi, F. Santo Pedro, L. C. de Barros, W. Lodwick, Fréchet derivative for linearly correlated fuzzy function, Information Sciences 435 (2018) 150-160.

[16] E. Kreyszig, Introductory functional analysis with applications, Vol. 1, wiley New York, 1978.

[17] B. Laiate, F. Santo Pedro, E. Esmi, L. C. de Barros, Hiv dynamics under antiretroviral treatment with interactivity, in: International Conference on Information Processing and Management of Uncertainty in Knowledge-Based Systems, Springer, 2020, pp. 212-225.
[18] B. Laiate, R. Watanabe, F. Santo Pedro, E. Esmi, L. C. de Barros, A cross-product of s-linearly correlated fuzzy numbers, in: IEEE International Conference on Fuzzy Systems (FUZZ-IEEE), IEEE, 2021, submitted.

[19] F. Longo, B. Laiate, F. Santo Pedro, E. Esmi, L. C. Barros, J. F. C. C. Meyer, A-cross product for autocorrelated fuzzy processes: the hutchinson equation, in: North American Fuzzy Information Processing Society Annual Conference, Springer, 2021, submitted.

[20] A. J. Lotka, Undamped oscillations derived from the law of mass action., Journal of the american chemical society 42 (8) (1920) 1595-1599.

[21] J. D. Murray, Mathematical biology: I. An introduction, Vol. 17, Springer Science \& Business Media, 2007.

[22] A. Ömer, O. Ömer, A prey predator model with fuzzy initial values, Hacettepe Journal of Mathematics and Statistics 41 (3) (2012) 387-395.

[23] M. S. Peixoto, L. C. Barros, R. C. Bassanezi, O. A. Fernandes, An approach via fuzzy systems for dynamics and control of the soybean aphid, in: 2015 Conference of the International Fuzzy Systems Association and the European Society for Fuzzy Logic and Technology (IFSAEUSFLAT-15), Atlantis Press, 2015.

[24] C. M. Pereira, M. S. Cecconello, R. C. Bassanezi, Prey-predator model under fuzzy uncertanties, in: North American Fuzzy Information Processing Society Annual Conference, Springer, 2018, pp. 408-418.

[25] F. Santo Pedro, L. C. de Barros, E. Esmi, Population growth model via interactive fuzzy differential equation, Information Sciences 481 (2019) 160-173.

[26] F. Santo Pedro, E. Esmi, L. C. de Barros, Calculus for linearly correlated fuzzy function using fréchet derivative and riemann integral, Information Sciences 512 (2020) 219-237.

[27] V. Volterra, Variazioni e fluttuazioni del numero d'individui in specie animali conviventi.

[28] L. A. Zadeh, Fuzzy sets, Information and Control 8 (3) (1965) 338-353. 